\title{
Total aortic arch replacement with a branched graft and limited circulatory arrest of the brain
}

Nicholas T. Kouchoukos, MD

Paolo Masetti, MD
From the Division of Cardiovascular and Thoracic Surgery, Department of Surgery, Missouri Baptist Medical Center, St Louis, Mo.

Supported in part by a grant to the Missouri Baptist Healthcare Foundation from Boston Scientific Inc, Natick, Mass.

Received for publication Aug 25, 2003; revised Nov 27, 2003; accepted for publication Dec 12, 2003.

Address for reprints: Nicholas T. Kouchoukos, MD, Missouri Baptist Medical Center, Suite 266 C, 3009 N Ballas Rd, St Louis, MO 63131 (E-mail: NTKouch@aol.com).

J Thorac Cardiovasc Surg 2004;128:233-7

$0022-5223 / \$ 30.00$

Copyright $\odot 2003$ by The American Association for Thoracic Surgery

doi:10.1016/j.jtcvs.2003.12.031
Background: Total replacement of the aortic arch is commonly performed with either antegrade perfusion of the brachiocephalic arteries by means of direct cannulation or with an interval of hypothermic circulatory arrest of at least 30 to 40 minutes. We present a technique with a branched graft that uses antegrade brain perfusion without the need for direct cannulation of the brachiocephalic arteries or a separate perfusion circuit, with only a brief period of circulatory arrest of the brain.

Methods: Twelve patients underwent resection of the aortic arch through either a midline sternotomy (4 patients) or a bilateral anterior thoracotomy (8 patients). The right axillary artery was used for arterial return and for brain perfusion. After establishing hypothermic circulatory arrest, the brachiocephalic arteries were detached from the aorta, flushed, and occluded with clamps. Hypothermic perfusion of the brain was established through the right axillary artery, and the brachiocephalic arteries were sequentially attached to the limbs of a branched aortic graft. Flow to the brain was then established in the antegrade direction through the axillary artery.

Results: The mean duration of circulatory arrest of the brain at a mean nasopharyngeal temperature of $16^{\circ} \mathrm{C}$ was 8.8 minutes (range, 6-13 minutes). The subsequent period of hypothermic $\left(20^{\circ} \mathrm{C}-22^{\circ} \mathrm{C}\right)$ brain perfusion, during which the 3 branches of the graft were attached to the brachiocephalic arteries, averaged 35 minutes (range, 23-44 minutes). All the patients survived the procedure and were discharged from the hospital. No patient sustained a permanent neurologic deficit. One patient had lethargy for 2 days, with full recovery. Nine of the 12 patients were extubated within 72 hours.

Conclusions: This technique obviates the need for direct cannulation of the brachiocephalic arteries and for a separate perfusion circuit and requires only a brief period of circulatory arrest of the brain.

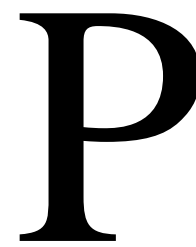
ermanent or transient neurologic dysfunction remains a major complication of operations that involve total replacement of the aortic arch. Currently, 2 general methods of brain protection are used with these extensive surgical procedures: profound hypothermic circulatory arrest with or without retrograde brain perfusion and selective antegrade hypothermic perfusion of the brachiocephalic arteries. Hypothermic circulatory arrest provides a dry operative field, but the safe duration of arrest is finite and should not exceed 45 to 50 minutes. Antegrade brain perfusion is usually accomplished by means of direct cannulation of the brachiocephalic arteries. This technique requires a separate perfusion circuit and careful monitoring of perfusion pressure and flow and introduces the risk of embolization of atheromatous debris or air.

We present our technique for total arch replacement using right axillary artery cannulation, a branched aortic graft, a short (less than 13 minutes) interval of circulatory arrest of the brain, and subsequent hypothermic brain perfusion but 


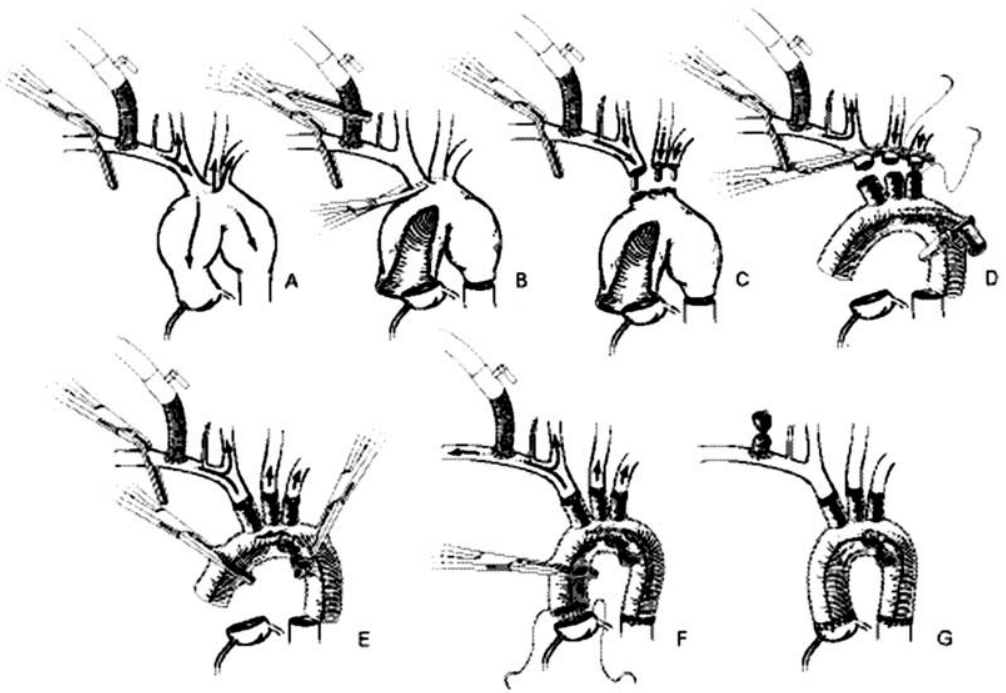

Figure 1. Technique for single-stage replacement of the thoracic aorta with a bilateral anterior thoracotomy incision: the arch-first technique and a branched aortic graft (see text for details).

without the need for direct cannulation of the brachiocephalic arteries or a separate perfusion circuit.

\section{Methods}

A median sternotomy is used for replacement of the ascending aorta, arch, and proximal descending thoracic aorta. If more extensive resection of the descending thoracic aorta is required, a bilateral anterior thoracotomy (clamshell incision) through the fourth intercostal spaces is used. ${ }^{1}$ With the latter approach, the right axillary artery is exposed through a subclavicular incision, and an 8-or 10-mm collagen-impregnated polyester graft (Meadox Hemashield Platinum 4 Branch Graft; Boston Scientific Inc, Natick, Mass) is sutured to the artery in an end-to-side fashion (Figure 1, A). The distal axillary artery is occluded when technically feasible to avoid hyperperfusion of the right arm. Arterial pressure is monitored in the left arm. A single, 2-stage venous cannula is inserted into the right atrium directly or through the right femoral vein. After establishing cardiopulmonary bypass (CPB), cooling is initiated. The left heart is vented with a catheter inserted in the right superior pulmonary vein, and a cannula is inserted into the coronary sinus for delivery of cold blood cardioplegic solution.

The head is packed in ice, methylprednisolone $(7-10 \mathrm{mg} / \mathrm{kg})$ and thiopental (10-15 mg/kg) are administered intravenously, and when the nasopharyngeal temperature reaches $13^{\circ} \mathrm{C}$ to $18^{\circ} \mathrm{C}$ and the electroencephalogram becomes isoelectric, the patient is placed in the Trendelenburg position, and circulatory arrest is established. A clamp is placed on the axillary perfusion graft, and the ascending aorta and aortic arch are opened, taking care to avoid dislodgment of atheromatous debris (Figure 1,B). The brachiocephalic arteries are dissected from the surrounding tissue, and the arteries are transected at their origins from the aorta (Figure $1, B$ ). If necessary, they are divided more distally to avoid areas of atheroma or dissection. Perfusion from the axillary artery is slowly initiated to evacuate entrapped air (Figure 1,C). The arteries are then clamped, and flow $(10-15 \mathrm{~mL} / \mathrm{kg})$ at a temperature of $20^{\circ} \mathrm{C}$ to $22^{\circ} \mathrm{C}$ is established to the brain by means of the axillary graft through the right carotid and right vertebral arteries (Figure 1, D). The flow rate is adjusted to maintain a mixed venous oxygen saturation measured in the right atrium of $85 \%$ to $95 \%$. Perfusion pressure is also monitored by using the arterial line in the left arm. The divided brachiocephalic arteries are attached to the limbs of an aortic branched graft (Meadox Hemashield Platinum 4 Branch Graft, Boston Scientific Inc), beginning with the left subclavian artery and ending with the innominate artery, with 5-0 polypropylene sutures (Figure 1,D). When the anastomoses are completed, the aortic graft is clamped distal to the left subclavian artery, the clamps on the 3 branches are removed, and air is evacuated from the proximal open end of the aortic graft. The aortic graft is then clamped just proximal to the innominate artery, and antegrade flow is established through the 3 arteries, maintaining the same flow rate, pressure, and temperature (Figure 1,E). The fourth branch of the aortic graft is ligated, and the graft is anastomosed to the descending thoracic aorta at the appropriate level (Figure 1, F). Rewarming is then initiated, perfusate flow is increased, and the anastomosis of the aortic graft to the ascending aorta is completed (Figure 1, G). Aortic valve or root replacement and coronary artery bypass grafting, if indicated, are performed at this time. CPB is discontinued after rewarming has been completed, and the axillary artery graft is ligated close to the artery and divided.

If resection of only the very proximal portion of the descending thoracic aorta is required, the procedure is performed through a median sternotomy. In most instances extension of the incision into the neck or division of the innominate vein is not necessary. $\mathrm{CPB}$ is established with perfusion through the axillary artery, as previously described (Figure 2, A). After circulatory arrest is established, the ascending aorta and arch are opened, and the 


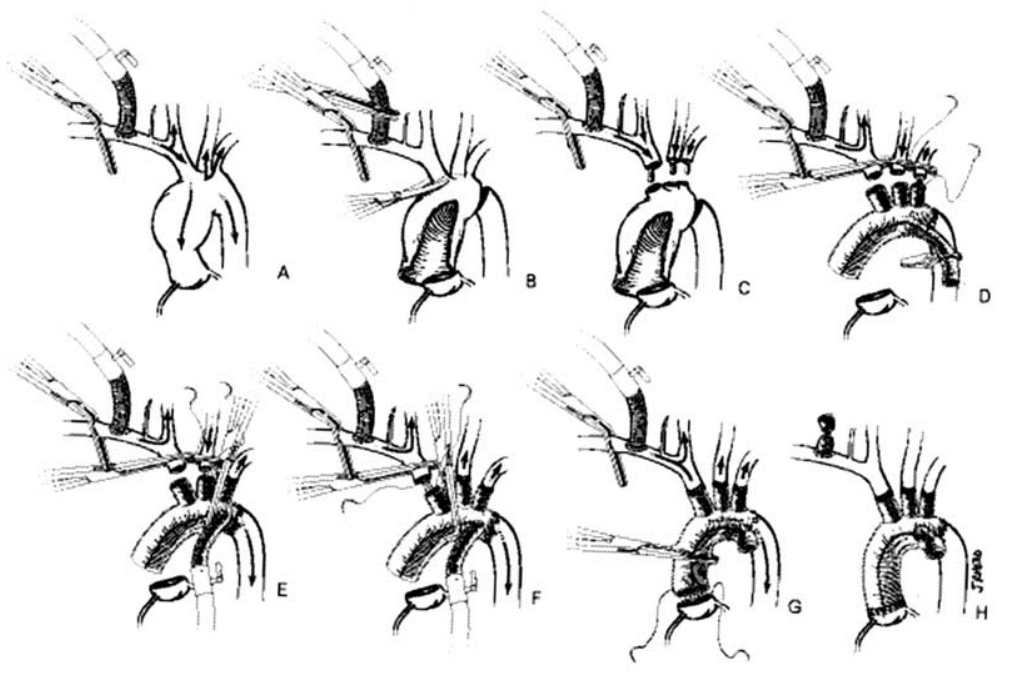

Figure 2. Technique for total arch replacement with a median sternotomy incision and a branched aortic graft (see text for details).

brachiocephalic arteries are transected (Figure 2, B). Perfusion from the axillary artery is slowly initiated to evacuate air (Figure $2, C)$, the arteries are clamped, and flow is established to the brain through the right vertebral and right carotid arteries (Figure 2,D). The proximal descending thoracic aorta is mobilized and divided, and the branched aortic graft is anastomosed first to the descending thoracic aorta (Figure 2, D). If indicated, the elephant trunk technique can be used for this anastomosis. The left subclavian artery is then anastomosed to the most distal branch of the aortic graft (Figure 2, D). After this anastomosis is completed, a second arterial line from the pump oxygenator is attached to the fourth branch of the aortic graft, and after evacuation of air, flow is established to the distal aorta and to the left subclavian artery. A clamp is placed on the aortic graft just proximal to this area (Figure $2, E$ ). Flow is simultaneously reduced through the right axillary artery to maintain total flow through the brachiocephalic arteries at the appropriate level $(10-15 \mathrm{~mL} / \mathrm{kg})$. The mixed venous oxygen saturation in the right atrium and the arterial pressure in the left arm are monitored. A single pump head of the CPB apparatus is used, and the desired ratio of flow through the 2 arterial lines is achieved by using occluders and inline flowmeters. The anastomosis of the middle branch of the aortic graft to the left carotid artery is then completed, and after evacuation of air and repositioning of the clamp on the graft proximal to the second branch, flow is established in an antegrade direction to the left carotid artery (Figure 2, F). Flow in the 2 arterial lines is again adjusted, and rewarming is begun. The anastomosis of the first branch of the graft to the innominate artery is then completed, maintaining antegrade flow into the left vertebral and left carotid arteries (Figure 2,F). After evacuation of air, the clamp on the aortic graft is positioned proximal to the first branch, and antegrade flow is established through all 3 brachiocephalic arteries and to the distal aorta through the right axillary artery (Figure 2, G). Flow through the fourth branch of the graft is discontinued, and this branch is ligated and divided (Figure 2, $H$ ). The procedure is then completed as described above.

\section{Results}

These techniques have been used in 12 patients undergoing total aortic arch replacement. Their ages ranged from 33 to 79 years (mean, 60 years). Eight patients had extensive thoracic aortic replacements with a bilateral anterior thoracotomy, and 4 had ascending aortic and total arch replacement with a median sternotomy. No patient had acute aortic dissection, 5 patients had chronic expanding aortic dissections, and 7 had degenerative aneurysms. Circulatory arrest of the brain was established at a mean temperature of $16^{\circ} \mathrm{C}$ (range, $13^{\circ} \mathrm{C}-18^{\circ} \mathrm{C}$ ), and the mean duration of arrest was 8.8 minutes (range, 6-13 minutes). The subsequent period of hypothermic perfusion during which the 3 branches of the graft were attached to the brachiocephalic arteries averaged 35 minutes (range, 23-44 minutes).

The mean durations of cooling, rewarming, and CPB were $35 \pm 7,68 \pm 18$, and $181 \pm 48$ minutes, respectively. There were no significant differences for these times between the 8 patients in whom a bilateral anterior thoracotomy was used and the 4 patients who had a median sternotomy. The mean durations of lower body circulatory arrest were $58 \pm 20$ and $30 \pm 13$ minutes for the above 2 groups, respectively $(P=.05)$. The difference was related to the more extensive descending thoracic aortic resections that were required in the patients having a bilateral anterior thoracotomy.

All patients survived the procedure and were discharged from the hospital. Six of the 12 patients were extubated within 24 hours, and 9 were extubated within 72 hours. One patient had lethargy for 48 hours, with complete recovery. No patient sustained a permanent neurologic deficit. The median and mean lengths of stay in the intensive care unit were 4 and 4.5 days, respectively. 


\section{Discussion}

The optimal technique for brain protection during operations that require total aortic arch replacement has not been clearly established. Antegrade cerebral perfusion with or without use of a branched graft is increasingly being used. ${ }^{2}$ This technique reduces the interval of circulatory arrest but introduces the risk of embolization of air or atherosclerotic debris from direct cannulation and perfusion of the brachiocephalic arteries and the potential risk for hyperperfusion or hypoperfusion. It generally requires use of a separate perfusion circuit to control pressure and flow in these arteries. Other techniques that avoid direct cannulation of the brachiocephalic arteries require intervals of circulatory arrest of at least 25 to 30 minutes to perform the anastomosis between the cuff of aorta surrounding the brachiocephalic arteries and the aortic graft or the individual anastomoses of the brachiocephalic arteries to the limbs of the branched graft. ${ }^{3,4}$ The technique we describe avoids cannulation of the brachiocephalic arteries and requires only a brief $(<13$ minutes; mean, 8.8 minutes) interval of circulatory arrest of the brain. Subsequent hypothermic perfusion of the brain from the right carotid and right vertebral arteries is achieved by means of perfusion through the right axillary artery cannula. The technique is applicable to procedures performed through either a median sternotomy or through more extensive incisions when replacement of part or all of the descending thoracic aorta is required in addition to aortic arch and ascending aortic replacement.

A potential limitation of this technique is inadequate protection of the left hemisphere because of insufficient collateral circulation between the right and left carotid and vertebral arterial systems. Frist and colleagues ${ }^{5}$ used direct perfusion of only the innominate or left carotid artery with moderate hypothermia $\left(26^{\circ} \mathrm{C}-28^{\circ} \mathrm{C}\right)$ and reported no temporary or permanent neurologic deficits in a series of 10 patients. Dossche and associates ${ }^{6}$ used unilateral antegrade cerebral perfusion in 37 patients and bihemispheric antegrade perfusion in 69 patients undergoing operations on the proximal thoracic aorta. In their multivariate analysis, the type of perfusion was not a predictor of temporary or permanent neurologic dysfunction. They also reported that among patients in their institution undergoing isolated unilateral carotid endarterectomy, an incomplete or absent circle of Willis was identified preoperatively only in $8 \%$ of cases.

Two additional studies using selective perfusion of only the innominate artery for aortic arch replacement have documented the safety of this technique. Aoyagi and cowork$\mathrm{ers}^{7}$ reported no neurologic complications in 11 patients. They used the distal stump pressure in the left superficial temporal artery to assess the patency of the circle of Willis. At a rectal temperature of $20^{\circ} \mathrm{C}$ to $23^{\circ} \mathrm{C}$ and with absence of activity on the electroencephalogram, perfusion through the right axillary artery was established at a flow rate of $10 \mathrm{~mL}$ $\cdot \mathrm{kg}^{-1} \cdot \mathrm{min}^{-1}$. The proximal innominate, left carotid, and left subclavian arteries were occluded. The duration of brachiocephalic perfusion was $57.6 \pm 15.1$ minutes. In no instance was perfusion of the left hemisphere judged to be inadequate. Wozniak and colleagues ${ }^{8}$ used selective cerebral perfusion through the innominate artery alone with moderate hypothermia $\left(28^{\circ} \mathrm{C}\right)$ in 25 consecutive patients. They used a test interval of 10 minutes of perfusion with monitoring of cerebral blood flow by using pulsed Doppler transcranial ultrasonography and bilateral somatosensoryevoked potentials. No patient was considered unsuitable for selective cerebral perfusion as a result of the test perfusion, and no patient had a new neurologic deficit postoperatively. Taken together, these studies suggest that hypothermic perfusion of the brain through the right axillary or right innominate artery provides sufficient protection of both cerebral hemispheres during replacement of the aortic arch.

It is possible, although unlikely, that hypoperfusion of the left side of the brain occurred during the period of hypothermic perfusion in our patients. In our series no extracranial occlusive disease of the carotid, subclavian, or vertebral arteries was demonstrated by means of preoperative angiography. During the interval of brain perfusion through the right axillary artery, which averaged 34 minutes, the temperature of the perfusate was maintained between $20^{\circ} \mathrm{C}$ and $22^{\circ} \mathrm{C}$, and the head was packed in ice. The combined duration of circulatory arrest and hypothermic perfusion of the brain did not exceed 53 minutes. This interval is within the range of the duration of hypothermic circulatory arrest for operations that included aortic arch replacement reported in several large studies in which hypothermic circulatory arrest was the sole method used for cerebral protection. ${ }^{9,10}$ The absence of any permanent neurologic deficit and only one minor transient deficit in our patients suggests that brain perfusion was adequate.

We have not used this technique in patients with acute type A aortic dissection. We believe, however, that it could be used in such patients if the dissection does not compromise flow into the innominate artery. On the basis of this initial experience, we believe that continued evaluation of this technique is warranted.

\section{References}

1. Rokkas CK, Kouchoukos NT. Single-stage extensive replacement of the thoracic aorta: the arch-first technique. J Thorac Cardiovasc Surg. 1999;117:99-105.

2. Kazui T, Yamashita K, Washiyama N, Terada H, Bashar AHM, Suzuki T, et al. Usefulness of antegrade selective cerebral perfusion during aortic arch operations. Ann Thorac Surg. 2002;74(suppl): S1806-9.

3. Spielvogel D, Mathur MN, Lansman SL, Griepp RB. Aortic arch reconstruction using a trifurcated graft. Ann Thorac Surg. 2003;75: 1034-6.

4. Nishimura M, Ohtake S, Sawa Y, Takahashi T, Matsumiya G, 
Kagisaki K, et al. Arch-first technique for aortic arch aneurysm repair through median sternotomy. Ann Thorac Surg. 2002;74:1264-6.

5. Frist WH, Baldwin JC, Starnes VA, Stinson EB, Oyer PE, Miller DC, et al. A reconsideration of cerebral perfusion in aortic arch replacement. Ann Thorac Surg. 1986;42:273-81.

6. Dossche KM, Schepens MAAM, Morshuis WJ, Muysoms FE, Langemeijer JJ, Vermeulen FEE. Antegrade selective cerebral perfusion in operations on the proximal thoracic aorta. Ann Thorac Surg. 1999;67: 1904-10.

7. Aoyagi S, Akashi H, Kubota Y, Momosaki M, Suzuki S, Oryoji A, et al. Partial brachiocephalic perfusion in aortic arch replacement. Surg Today. 1993;23:331-7.
8. Wozniak G, Dapper F, Schindler E, Akinturk H, Zickmann B, Gehron $\mathrm{J}$, et al. An assessment of selective cerebral perfusion via the innominate artery in aortic arch replacement. Thorac Cardiovasc Surg. 1998;46:7-11.

9. Grabenwoger M, Ehrlich M, Cartes-Zumelzu F, Mittlbock M, Weigel G, Laufer G, et al. Surgical treatment of aortic arch aneurysms in profound hypothermia and circulatory arrest. Ann Thorac Surg. 1997; 64:1067-71.

10. Hagl C, Ergin MA, Galla JD, Lansman SL, McCullogh JN, Spielvogel $\mathrm{D}$, et al. Neurologic outcome after ascending aorta-aortic arch operations: effect of brain protection technique in high-risk patients. $J$ Thorac Cardiovasc Surg. 2001;121:1107-21.

\section{ON THE MOVE?}

Don't miss a single issue of the journal! To ensure prompt service when you change your address, please photocopy and complete the form below.

Please send your change of address notification at least six weeks before your move to ensure continued service. We regret we cannot guarantee replacement of issues missed due to late notification.

\section{JOURNAL TITLE:}

Fill in the title of the journal here.

\section{OLD ADDRESS:}

Affix the address label from a recent issue of the journal here.

\section{NEW ADDRESS:}

Clearly print your new address here.

Name

Address

City/State/ZIP
COPY AND MAIL THIS FORM TO:

Elsevier Inc.

Subscription Customer Service

6277 Sea Harbor Dr

Orlando, FL 32887
OR FAX TO:

407-363-9661

OR E-mail:

elspcs@elsevier.com
OR PHONE:

800-654-2452

Outside the U.S., call

407-345-4000 